Tôhoku Math. Journ.

22(1970), 130-137.

\title{
ESTIMATES FOR THE MAXIMAL FUNCTION OF HARDY-LITTLEWOOD AND THE MAXIMAL HILBERT TRANSFORM WITH WEIGHTED MEASURES
}

\author{
MAKOTO KANEKO
}

(Received September 26, 1969)

Introduction. When we consider the Hilbert transform $\widetilde{f}(x)=\mathrm{v} \cdot \mathrm{p} . \int_{-\infty}^{\infty} \frac{f(t)}{x-t} d t$ of a function $f$, we have to treat the function $\widetilde{f}_{s}(x)=\int_{|x-t| \geqq s} \frac{f(t)}{x-t} d t$. In particular, it is interesting to estimate the maximal Hilbert transform $\widetilde{f^{*}}(x)$ $=\sup _{\epsilon}\left|\widetilde{f}_{\odot}(x)\right|$ by the measure $m(e)=\int_{e} \frac{1}{\delta+|t|^{\alpha}} d t$, where $\delta=0$ or 1 and $0 \leqq \alpha<1$. S. Koizumi [4] shows that the operator $f \rightarrow \widetilde{f}$ is of weak type $(1,1)$ with respect to the measure $m(e)$ where $\delta=1$. He says also that the operator $f \rightarrow \widetilde{f}^{*}$ is of weak type $(1,1)$ with respect to the same measure and the proof is carried over by the same method. However the latter proposition does not seem to be proved as the former proposition ${ }^{13}$. The purpose of this paper is to give the complete proof of this proposition.

We estimate the maximal function of Hardy-Littlewood with respect to the measure $m(e)$ in $\$ 1$ and then $\tilde{f}^{*}$ in $\$ 2$ with the same measure.

I wish to express my gratitude to Messrs. G. Sunouchi, S. Igari and K. Yabuta for guidance and encouragement during preparation of this paper.

1. Maximal function of Hardy-Littlewood. For a non-negative locally integrable function $f$ on $(-\infty,+\infty)$, the maximal function is defined by

$$
(\Theta f)(x)=\sup _{\varsigma} \frac{1}{\varepsilon} \int_{|x-t| \leqq \varsigma} f(t) d t
$$

where $d t$ is the Lebesgue measure, $d m$ is a measure on $(-\infty,+\infty)$ defined by

$$
m(e)=\int_{e} \frac{1}{\delta+|t|^{\alpha}} d t, \quad 0 \leqq \alpha<1, \delta=0 \text { or } 1
$$

1) For example, see Y.M. Chen [1], in particular, p. 243 footnote. 
and $L_{m}^{p}$ represents the set of all functions such that $\int_{-\infty}^{\infty}|f(t)|^{p} d m<+\infty$.

In the following, $c$ denotes a constant depending only on $\alpha$, and may be different in each occurence.

THEOREM 1. If a non-negative function $f$ belongs to $L_{m}^{1}$, we have for any $\lambda>0$

$$
m(\{x ;(\Theta f)(x)>\lambda\}) \leqq \frac{c}{\lambda} \int_{-\infty}^{\infty} f(t) d m(t)
$$
$\theta f:$

To prove this theorem, it is sufficient to show the following proposition on

$$
(\theta f)(x)=\sup _{-\infty<\xi<x} \frac{1}{x-\xi} \int_{\xi}^{x} f(t) d t
$$

PROPOSITION 1. If a non-negative function $f$ belongs to $L_{m}^{1}$, we have for any $\lambda>0$.

$$
m(\{x ;(\theta f)(x)>\lambda\}) \leqq \frac{c}{\lambda} \int_{-\infty}^{\infty} f(t) d m(t)
$$

PROOF. Since

$$
\frac{1}{x-\xi} \int_{\xi}^{x} f(t) d t=\frac{1}{m(\xi, x)} \int_{\xi}^{x} \frac{m(\xi, x)\left(\delta+|t|^{\alpha}\right)}{x-\xi} f(t) d m(t)
$$

where $m(\xi, x)$ means the $m$-measure $m([\xi, x])$ of the interval $[\xi, x]$, let us estimate

$$
I=\frac{m(\xi, x)\left(\delta+|t|^{\alpha}\right)}{x-\xi}
$$

under the condition $\xi<t \leqq x$.

(1) Case $0 \leqq \xi<x$. Since $1 /\left(\delta+S^{a}\right)$ is decreasing in $0<S<+\infty$,

$$
I=\frac{\delta+t^{\alpha}}{x-\xi} \int_{\xi}^{x} \frac{d S}{\delta+S^{\alpha}} \leqq \frac{\delta+t^{\alpha}}{x} \int_{0}^{x} \frac{d S}{\delta+S^{\alpha}} \leqq \frac{\delta+x^{\alpha}}{x} \int_{0}^{x} \frac{d S}{\delta+S^{\alpha}}
$$

(2) Case $\xi<x \leqq 0$. Similarly to (1), we have 


$$
I \leqq \frac{\delta+|\xi|^{\alpha}}{|\xi|} \int_{0}^{|\xi|} \frac{d S}{\delta+S^{\alpha}}
$$

(3) Case $\xi<0<x$. Setting $N=\operatorname{Max}(|\xi|, x)$,

$$
I=\frac{\delta+|t|^{\alpha}}{x-\xi}\left(\int_{\xi}^{0}+\int_{0}^{x}\right) \frac{d S}{\delta+|S|^{\alpha}} \leqq \frac{2\left(\delta+N^{\alpha}\right)}{N} \int_{0}^{N} \frac{d S}{\delta+S^{\alpha}} .
$$

Therefore in any cases,

$$
\frac{m(\xi, x)\left(\delta+|t|^{\alpha}\right)}{x-\xi} \leqq \frac{2\left(\delta+N^{\alpha}\right)}{N} \int_{0}^{N} \frac{d S}{\delta+S^{\alpha}} \leqq c
$$

Consequently we get

$$
(\theta f)(x) \leqq c \sup _{-\infty<\xi<x} \frac{1}{m(\xi, x)} \int_{\xi}^{x} f(t) d m(t)
$$

Thus we shall have Proposition 1, if we show the next proposition on $\Lambda f$ :

$$
(\Lambda f)(x)=\sup _{-\infty<\xi<x} \frac{1}{m(\xi, x)} \int_{\xi}^{x} f(t) d m(t) .
$$

PROPOSITION 2. If a non-negative function $f$ belongs to $L_{m}^{1}$, we have for any $\lambda>0$

$$
m(\{x ;(\Lambda f)(x)>\lambda\}) \leqq \frac{c}{\lambda} \int_{-\infty}^{\infty} f(t) d m(t) .
$$

This is a consequence of the Theorem 2.1. in [2].

REMARK. Theorem 1 is false for $\alpha>1$. Let $f(t)=t^{\beta}$ for $t>1$ and $f(t)=0$ for $t \leqq 1$, where $0<\beta<\alpha-1$, then we find that $(\Theta f)(x)=+\infty$ for all $x$. Thus we get that $m(\{x ;(\Theta f)(x)>\lambda\})=$ constant $(\delta=1),=\infty(\delta=0)$, which is impossible.

\section{Maximal Hilbert transform.}

THEOREM 2. If a function $f$ belongs to $L_{m}^{1}$, we have for any $\lambda>0$ 


$$
m\left(\left\{x ; \widetilde{f}^{*}(x)>\lambda\right\}\right) \leqq \frac{c}{\lambda} \int_{-\infty}^{\infty}|f(t)| d m(t) .
$$

In order to prove this theorem, we start with the following lemma which is stated in [3] when $\delta=0$ and, when $\delta=1$, it can be shown similarly.

LemMa 1. For any $f \in L_{m}^{1}$ and $\lambda>0$, we get the following decomposition:

$$
f(t)=v(t)+\sum_{n} w_{n}(t)=v(t)+w(t)
$$

(2.3) $I_{n}$ do not contain the origin in their insides and are mutually disjoint,

$$
\begin{gathered}
|v(t)| \leqq c \lambda, \\
\int_{-\infty}^{\infty}|v(t)| d m(t)+\sum_{n} \int_{-\infty}^{\infty}\left|w_{n}(t)\right| d m(t) \leqq c \int_{-\infty}^{\infty}|f(t)| d m(t) \\
\sum_{n} m\left(I_{n}\right) \leqq \frac{1}{\lambda} \int_{-\infty}^{\infty}|f(t)| d m(t) \\
\int_{-\infty}^{\infty} w_{n}(t) d t=0
\end{gathered}
$$

Corresponding the above decomposition, we get $\widetilde{f}^{*}(x) \leqq \widetilde{v}^{*}(x)+\widetilde{r}^{*}(x)$. We see $v \in L_{m}^{2}$ by virtue of (2.4) and (2.5). By [5] we get

$$
\int_{-\infty}^{\infty} \widetilde{v}^{*}(x)^{2} d m(x) \leqq c \int_{-\infty}^{\infty}|v(t)|^{2} d m(t) .
$$

So we get, taking (2.4) in consideration,

$$
m\left(\left\{x ; \widetilde{v}^{*}(x)>\frac{\lambda}{2}\right\}\right) \leqq \frac{c}{\lambda} \int_{-\infty}^{\infty}|f(t)| d m(t) .
$$

Next we turn our attention to $\widetilde{w}^{*}(x)$. We denote by $a_{n}$ the center of $I_{n}$ in the Lemma 1, and $I_{n}^{*}$ the interval which is obtained by magnifying $I_{n}$ two tịmes with center $a_{n}$ and set $Q^{*}=\bigcup_{n} I_{n}^{*}$. Let us investigate $\widetilde{w}_{n \bullet}(x)$ for $x$ in 
$C Q^{*}$.

Setting $B(x, \varepsilon)=\{t ;|x-t| \leqq \varepsilon\}$, we get by (2.7)

$$
\begin{aligned}
\widetilde{w}_{n s}(x) & =\int_{|x-t| \geqq c} \frac{w_{n}(t)}{x-t} d t \\
& = \begin{cases}\int_{-\infty}^{\infty}\left(\frac{1}{x-t}-\frac{1}{x-a_{n}}\right) w_{n}(t) d t, & \text { if } I_{n} \cap B(x, \varepsilon)=\emptyset \\
\int_{|x-t| \geqq c} \frac{w_{n}(t)}{x-t} d t, & \text { if } I_{n} \cap B(x, \varepsilon) \neq \emptyset .\end{cases}
\end{aligned}
$$

If $I_{n} \cap B(x, \varepsilon) \neq \emptyset, \quad I_{n} \subset B(x, 3 \varepsilon)$, so that

$$
\int_{|x-t| \geqq \varepsilon} \frac{\left|w_{n}(t)\right|}{|x-t|} d t \leqq \frac{1}{\varepsilon} \int_{-\infty}^{\infty}\left|w_{n}(t)\right| d t=\frac{1}{\varepsilon} \int_{|x-t| \leqq 3 \varepsilon}\left|w_{n}(t)\right| d t,
$$

and that we find

$$
\left|\widetilde{w}_{n \varepsilon}(x)\right| \leqq \int_{-\infty}^{\infty}\left|\frac{1}{x-t}-\frac{1}{x-a_{n}}\right|\left|w_{n}(t)\right| d t+\frac{1}{\varepsilon} \int_{|x-t| \leqq 3 \varepsilon}\left|w_{n}(t)\right| d t
$$

Summing up with respect to $n$, we get

$$
\begin{aligned}
\left|\widetilde{w}_{c}(x)\right| & \leqq \sum_{n} \int_{-\infty}^{\infty}\left|\frac{1}{x-t}-\frac{1}{x-a_{n}}\right|\left|w_{n}(t)\right| d t+\frac{1}{\varepsilon} \int_{|x-t| \leqq s s}|w(t)| d t \\
& \leqq \sum_{n} \int_{-\infty}^{\infty}\left|\frac{1}{x-t}-\frac{1}{x-a_{n}}\right|\left|w_{n}(t)\right| d t+3(\Theta w)(x),
\end{aligned}
$$

where $\Theta w$ stands for $\Theta(|w|)$. Consequently,

$$
w^{*}(x) \leqq \sum_{n} \int_{-\infty}^{\infty}\left|\frac{1}{x-t}-\frac{1}{x-a_{n}}\right|\left|w_{n}(t)\right| d t+3(\Theta w)(x)
$$

for $x \in C Q^{*}$.

Hence

$$
\int_{C Q^{*}}\left(\sum_{n} \int_{-\infty}^{\infty}\left|\frac{1}{x-t}-\frac{1}{x-a_{n}}\right|\left|w_{n}(t)\right| d t\right) d m(x)
$$




$$
\leqq \sum_{n} \int_{C I_{n}^{*}} d m(x) \int_{-\infty}^{\infty}\left|\frac{1}{x-t}-\frac{1}{x-a_{n}}\right|\left|w_{n}(t)\right| d t
$$

By virtue of the following Lemma 2, the last sum does not exceed

$$
c \sum_{n} \int_{-\infty}^{\infty}\left|w_{n}(t)\right| d m(t)=c \int_{-\infty}^{\infty}|w(t)| d m(t)
$$

LEMMA 2. For a function $g$ whose support is contained in an interval $[a-k, a+k]$ not containing the origin in its inside, it holds that

$$
\int_{|x-a| \geqq 2 k} d m(x) \int_{-\infty}^{\infty}\left|\frac{1}{x-t}-\frac{1}{x-a}\right||g(t)| d t \leqq c \int_{-\infty}^{\infty}|g(t)| d m(t) .
$$

PROOF.

$$
\begin{aligned}
& \int_{|x-a| \geqq 2 k} d m(x) \int_{-\infty}^{\infty}\left|\frac{1}{x-t}-\frac{1}{x-a}\right||g(t)| d t \\
& =\int_{-k}^{k}|g(a+t)| d t \int_{|x| \geqq 2 k}\left|\frac{1}{x-t}-\frac{1}{x}\right| \frac{d x}{\delta+|x+a|^{\alpha}} \\
& =\int_{-k}^{k}|g(a+t)| d t\left(\int_{(|x| \geqq 2 k) \cap(|x+a| \leqq|a| / 2)}+\int_{(|x| \geqq 2 k) \cap(|x+a|>|a| / 2)}\right) \\
& \left|\frac{1}{x-t}-\frac{1}{x}\right| \frac{d x}{\delta+|x+a|^{\alpha}}
\end{aligned}
$$

For $|t| \leqq k$ and $x \in(|x| \geqq 2 k) \cap(|x+a|>|a| / 2),|a+t| \leqq|a|+|t|<2|a|$ $\leqq 4|x+a|$, so that $1 /\left(\delta+|x+a|^{\alpha}\right)<4 /\left(\delta+|t+a|^{\alpha}\right)$. We get

$$
\begin{aligned}
\int_{(|x| \geq 2 k) \cap(|x+a|>|a| / 2)}\left|\frac{1}{x-t}-\frac{1}{x}\right| \frac{d x}{\delta+|x+a|^{\alpha}} \leqq \frac{4}{\delta+|t+a|^{\alpha}} \int_{|x| \geqq 2 k}\left|\frac{1}{x-t}-\frac{1}{x}\right| d x \\
\leqq \frac{c}{\delta+|t+a|^{\alpha}} .
\end{aligned}
$$

While, if $\quad(|x| \geqq 2 k) \cap(|x+a| \leqq|a| / 2) \neq \emptyset$, then $k \leqq 3|a| / 4$, so that $|a+t|<2|a|, \quad$ and $|x-t| \geqq k$ and $|x| \geqq|a| / 2$ for $|t| \leqq k$ and 
$x \in(|x| \geqq 2 k \cap(|x+a| \leqq|a| / 2)$. So we get $|1 /(x-t)-1 / x| \leqq 2 /|a|$. Consequently, by (1.2)

$$
\begin{gathered}
\int_{(|x| \geqq 2 k) \cap(|x+a| \leqq|a| / 2)}\left|\frac{1}{x-t}-\frac{1}{x}\right| \frac{d x}{\delta+|x+a|^{\alpha}} \leqq \frac{2}{|a|} \int_{|x+a| \leqq|a| / 2} \frac{d x}{\delta+|x+a|^{\alpha}} \\
=\frac{2}{(|a| / 2)} \int_{0}^{|a| / 2} \frac{1}{\delta+|x|^{\alpha}} d x \leqq \delta+(|a| / 2)^{\alpha}<\frac{c}{\delta+(2|a|)^{\alpha}}<\frac{c}{\delta+|a+t|^{\alpha}} .
\end{gathered}
$$

Thus we get

$$
\begin{gathered}
\int_{|x-a| \geqq 2 k} d m(x) \int_{-\infty}^{\infty}\left|\frac{1}{x-t}-\frac{1}{x-a}\right||g(t)| d t \leqq c \int_{-k}^{k}|g(a+t)| \frac{1}{\delta+|a+t|^{\alpha}} d t \\
=c \int_{-\infty}^{\infty}|g(t)| d m(t) . \quad \text { (q. e. d.) }
\end{gathered}
$$

Since $\widetilde{w}^{*}(x)$ satisfies the inequality (2.8) for $x \in \mathcal{C} Q^{*}$,

$$
\begin{aligned}
\{x & \left.\in C Q^{*} ; w^{*}(x)>\frac{\lambda}{2}\right\} \\
& \subset\left\{x \in C Q^{*} ; \sum_{n} \int_{-\infty}^{\infty}\left|\frac{1}{x-t}-\frac{1}{x-a_{n}}\right|\left|w_{n}(t)\right| d t>\frac{\lambda}{4}\right\} \\
& \cup\left\{x \in C Q^{*} ;(\Theta w)(x)>\frac{\lambda}{12}\right\} \equiv E_{1} \cup E_{2}, \text { say } .
\end{aligned}
$$

From (2.9), we get

$$
m\left(E_{1}\right) \leqq \frac{c}{\lambda} \int_{-\infty}^{\infty}|w(t)| d m(t) \leqq \frac{c}{\lambda} \int_{-\infty}^{\infty}|f(t)| d m(t)
$$

While, from Theorem 1 ,

$$
m\left(E_{2}\right) \leqq \frac{c}{\lambda} \int_{-\infty}^{\infty}|w(t)| d m(t) \leqq \frac{c}{\lambda} \int_{-\infty}^{\infty}|f(t)| d m(t)
$$

Sọ that, if we shọ that $m\left(Q^{*}\right) \leqq \frac{c}{\lambda} \int_{7 \infty}^{\infty}|f(t)| d m(t)$, then we get 


$$
m\left(\left\{x ; \widetilde{w^{*}}(x)>\frac{\lambda}{2}\right\}\right) \leqq \frac{c}{\lambda} \int_{-\infty}^{\infty}|f(t)| d m(t)
$$

and the proof of Theorem 2 is completed. To prove that $m\left(Q^{*}\right) \leqq \frac{c}{\lambda} \int_{-\infty}^{\infty}$ $|f(t)| d m(t)$, it is sufficient to show $m\left(I_{n}^{*}\right) \leqq c m\left(I_{n}\right)$ due to (2.6). But this is clear from the following lemma.

LEMMA 3. If we put $I=(a-k, a+k), a>k>0, I^{*}=(a-2 k, a+2 k)$, then $m\left(I^{*}\right) \leqq c m(I)$.

PROOF. $m(a, a+2 k)=m(a, a+k)+m(a+k, a+2 k) \leqq m(a-k, a+k)$, so that we need only to show that $m(a-2 k, a) \leqq c m(a-k, a)$. But this comes from an elementary calculation; for example, consider the two cases $0<k<a / 4$ and $a / 4<k<a$.

\section{REFERENCES}

[1] Y.M. CHEN, Some asymptotic approximation methods. II, Proc. London Math. Soc., (3) 16(1966), 241-263.

[2] L. HớRMANDER, $L^{p}$ estimates for (pluri) subharmonic functions, Math. Scand., 20(1967), 65-78.

[ 3 ] S. IGARI, On the decomposition theorems of Fourier transforms with weighted norms, Tôhoku Math. J., 15(1963), 6-36.

[4] S. KoIzUMI, On the Hilbert transform I, J. of Faculty Science of Hokkaido University, series I, 14(1959), 153-224.

[5] P. KREE, Sur les multiplicateurs dans $\mathscr{F} L^{p}$ avec poids, Ann. Inst. Fourier(Grenoble), 16(1966), 91-121.

MATHEMATICAL INSTITUTE

TÔHOKU UNIVERSITY

SENDAI, JAPAN 